\title{
Correction to: Human adipose-derived stem cells partially rescue the stroke syndromes by promoting spatial learning and memory in mouse middle cerebral artery occlusion model
}

\author{
Fei Zhou ${ }^{1 \dagger}$, Shane $\mathrm{Gao}^{2 \dagger}$, Lin Wang ${ }^{3+}$, Chenxi Sun ${ }^{2}$, Lu Chen ${ }^{2}$, Ping Yuan ${ }^{4}$, Haiyang Zhao ${ }^{5}, Y_{i}$ Yi $^{1}$, Ying Qin ${ }^{2}$, \\ Zhiqiang Dong ${ }^{2}$, Limei Cao ${ }^{2}$, Haiyan Ren ${ }^{1}$, Liang Zhu ${ }^{2}$, Qiang Li ${ }^{1}$, Bing Lu ${ }^{2}$, Aibin Liang ${ }^{4}$, Guo-Tong Xu' \\ Hongwen $\mathrm{Zhu}^{7,8}$, Zhengliang Gao ${ }^{9,10}$, Jie $\mathrm{Ma}^{3^{*}}$, Jun $\mathrm{Xu}^{2^{*}}$ and Xu Chen ${ }^{1 *}$
}

\section{Correction to: Stem Cell Res Ther} https://doi.org/10.1186/s13287-015-0078-1

The original article [1] contains an accidental omission in the Acknowledgements. The corrected Acknowledgements section is shown ahead:

\section{Acknowledgements}

This work was supported by the Ministry of Science and Technology of China (2010CB945200, 2011CB966200); the National Natural Science Foundation of China (31471029, 81472141, 81070910, 81200832, 81271382); National program for support of Top-notch young professionals (J.X.); Shanghai City, the medical cooperation projects (11DZ192130C); Projects of International Cooperation and Exchanges NSFC (81261130318); the Fundamental Research Funds for the Central Universities; Tongji University Talents Training Program (2011KJ051); New Century Excellent Talents in University (NCET10-0606); 51th China Postdoctoral surface Project (2012 M510890); Shanghai Postdoctoral Research Funding Scheme of 2012 (12R21416200).

\begin{abstract}
Author details
'Neurology Department, Shanghai Eighth People's Hospital Affiliated to Jiangsu University, Shanghai 200233, China. ${ }^{2}$ East Hospital, Tongji University School of Medicine, Shanghai 200120, China. ${ }^{3}$ Department of Pediatric Neurosurgery, Xinhua Hospital of Shanghai Jiaotong University, Shanghai 200092, China. ${ }^{4}$ Tongji Hospital, Tongji University School of Medicine, Shanghai 200442, China. ${ }^{5}$ Shanghai Xu Hui District Hospital Affiliated to Jiangsu University, Shanghai 200031, China. '́Laboratory of Clinical Visual Science, Tongji Eye Institute, Tongji University School of Medicine, Shanghai 200092, China. ${ }^{7}$ Tianjin Hospital, Tianjin 300211, China. ${ }^{8}$ Tianjin Academy of Integrative Medicine, Tianjin 300100, China. Institute of Translational Medicine, Tongji University School of Medicine, Shanghai 200092, China. ${ }^{10}$ Tenth People's Hospital Affiliated to Tongji University, Shanghai 200092, China.
\end{abstract}

Received: 28 February 2019 Revised: 28 February 2019 Accepted: 28 February 2019 Published online: 06 March 2019

\section{Reference}

1. Zhou F, et al. Human adipose-derived stem cells partially rescue the stroke syndromes by promoting spatial learning and memory in mouse middle cerebral artery occlusion model. Stem Cell Res Ther. 2016;6:92 https://doi. org/10.1186/s13287-015-0078-1.

\footnotetext{
*Correspondence: majie365@hotmail.com; xunymc2000@yahoo.com; cxwp65@163.com

${ }^{\dagger}$ Fei Zhou, Shane Gao and Lin Wang contributed equally to this work. ${ }^{3}$ Department of Pediatric Neurosurgery, Xinhua Hospital of Shanghai Jiaotong University, Shanghai 200092, China

2East Hospital, Tongji University School of Medicine, Shanghai 200120, China

'Neurology Department, Shanghai Eighth People's Hospital Affiliated to

Jiangsu University, Shanghai 200233, China

Full list of author information is available at the end of the article
}

(c) The Author(s). 2019 Open Access This article is distributed under the terms of the Creative Commons Attribution 4.0 International License (http://creativecommons.org/licenses/by/4.0/), which permits unrestricted use, distribution, and reproduction in any medium, provided you give appropriate credit to the original author(s) and the source, provide a link to the Creative Commons license, and indicate if changes were made. The Creative Commons Public Domain Dedication waiver (http://creativecommons.org/publicdomain/zero/1.0/) applies to the data made available in this article, unless otherwise stated. 